NOT FOR QUOTATION

WITHOUT THE PERMISSION

OF THE AUTHOR

EPSHON SOLUTIONS AND DUALTY IN VECTOR OPTLMZATION

Istvăn Valyi

May 1987

WP-87-43

Working Papers are interim reports on work of the International Institute for Applied Systems Analysis and have received only limited review. Views or opinions expressed herein do not necessarily represent those of the Institute or of its National Member Organizations.

INTERNATIONAL INSTITUTE FOR APPLIED SYSTEMS ANALYSIS

2361 Laxenburg, Austria 


\section{PREFACE}

This paper is a continuation of the author's previous investigations in the theory of epsilon-solutions in convex vector optimization and serves as a theoretical background for the research of SDS in the field of multicriteria optimization. With the stress laid on duality theory, the results presented here give some insight into the problems arising when exact solutions have to be substituted by approximate ones. Just like in the scalar case, the available computational techniques frequently lead to such a situation in multicriteria optimization.

Alexander B. Kurzhanski

Chairman

Systems and Decision Sciences Area 


\section{CONTENTS}

1. Introduction 1

2. Epsilon Optimal Elements 3

3. Perturbation Map and Duality $\quad 7$

4. Conical Supports 12

$\begin{array}{lr}\text { 5. Conclusion } & 17\end{array}$

$\begin{array}{lr}\text { 6. References } & 18\end{array}$ 


\title{
EPSILON SOLUTIONS AND DUALITY IN VECTOR OPTIMIZATION
}

\author{
Istuan Valyi
}

\section{INTRODUCTION}

The study of epsilon solutions in vector optimization problems was started in 1979 by S. S. Kutateladze [1]. These types of solutions are interesting because of their relation to nondifferentiable optimization and the vector valued extensions of Ekeland's variational principle as considered by P. Loridan [2] and I. Vályi [3], but computational aspects are perhaps even more important. In practical situations, namely, we often stop the calculations at values that we consider sufficiently close to the optimal solution, or use algorithms that result in some approximates of the Pareto set. Such procedures can result in epsilon solutions that are under study in this paper. A paper by D. J. White [4] deals with this issue and investigates how well these solutions approximate the exact solutions.

Motivated by the above, in the present paper we study the implications in duality theory of substituting exact solutions with epsilon solutions. Although the well known results have their counterparts, our findings show that in some cases special caution is required.

For the sake of simplicity in formulation we shall restrict our consideration to finite dimensional spaces, although all the results have a corresponding version in infinite dimensions. Our major tool is the saddle point theorem for epsilon solutions and the techniques used in standard vector duality theory. For details see I. Valyi [5] and the book by Y. Sawaragi, H Nakayama and T. Tanino [6]. As a consequence of the fact that the notion of approximate solution coincides with that of exact solution in the case when the approximation error is zero, our results reduce to those related to exact vector optimization. From another point of view they are parallel to the theory of epsilon solutions in the scalar valued case as expounded 
e.g. by J. J. Strodiot, V. H. Nguyen and N. Heukemes [7], or in the vectorial case for absolute optimality by I. Vályi [8].

In this paper we give the proofs of the results presented at the VII-th International Conference on Multiple Criteria Decision Making, held between 18-22 August, 1986, in Kyoto, Japan. 


\section{EPSILON OPTIMAL ELEMENTS}

In this section we recall some basic notions and known facts (without proofs) related to $\varepsilon$-optimal solutions in vector optimization. All the vector spaces throughout the paper are real, finite dimensional and ordering cones are supposed to be convex, pointed, closed and to have a nonempty interior. $X, Y$ and $Z$ denote vector spaces while $C$ and $K$ are the positive cones of $Y$ and $Z$ respectively. The dual space of $Y$ is $Y$ and the cone of positive functionals with respect to $C \subset Y$, or the dual of $C$, is $C^{+}$and $L^{+}(Z, Y) \subset L(Z, Y)$ stands for the cone of positive linear maps from $Z$ to $Y$.

For the various ordering relationships between two elements of an ordered vector space we shall use the following notations, for example in $Y$ :

$$
\begin{array}{ccc}
y_{2} \geqq y_{1} \text { iff } & y_{2}-y_{1} \in C \\
y_{2} \geqq y_{1} \text { iff } & y_{2}-y_{1} \in C \backslash\{0\} \\
y_{2}>y_{1} \text { iff } & y_{2}-y_{1} \in \operatorname{int}(C)
\end{array}
$$

and $y_{2} \neq y_{1}$ will refer to the fact that $y_{1} \in Y$ does not dominate $y_{2} \in Y$ from below. Now for the readers convenience we quote the following definition from e. $g$. D. T. Luc [9].

Definition 2.1 .

The set $H \subset Y$ is $C$-convex if $H+C \subset Y$ is convex.

The function $f: X \rightarrow Y$ is $C$-convex if the set $\{f(x) \in Y: x \in d o m f\}$ is $C$-convex.

The set $H \subset Y$ is $C$-compact if there exists a bounded set $H_{0} \subset Y$ with the property $H \subset H_{0}+C$ and if $H+C \subset Y$ is closed.

Now turn to the consideration of $\varepsilon$-optimality. Throughout the paper the vectors $\varepsilon, \varepsilon_{n} \in C \subset Y$ will represent the approximation error and their value will be fixed.

Definition 2.2 .

The vector $y \in H$ is an $\varepsilon$-minimal element of $H \subset Y$, in notation $y \in \varepsilon-\min (H)$, if $(y-\varepsilon-C) \cap H \subset\{y-\varepsilon\}$.

it is weakly $\varepsilon$-minimal, in notation $y \in \varepsilon-w \min (H)$, if $(y-\varepsilon-\operatorname{int}(C)) \cap H C\{y-\varepsilon\}$ 
and properly $\varepsilon$-minimal, in notation $y \in \varepsilon-p \min (H)$, if there exists a $y^{*} \in \operatorname{int}\left(C^{+}\right)$ such that $\left\langle y^{*}, y-\varepsilon\right\rangle \leqq\left\langle y^{*}, h\right\rangle \quad \forall h \in H$.

The approximately maximal elements are to be defined in a corresponding manner.

The following statements are easy consequences of the definitions and clarify the relationships between the different notions of minimal element.

Proposition 2.1.

Suppose that $\varepsilon_{1} \leqq \varepsilon_{2}$. Then we have

$$
\begin{gathered}
\varepsilon_{1}-\min (H) \subset \varepsilon_{2}-\min (H) \\
\varepsilon_{1}-w \min (H) \subset \varepsilon_{2}-w \min (H) \\
\varepsilon_{1}-p \min (H) \subset \varepsilon_{2}-p \min (H)
\end{gathered}
$$

Proposition 2.2.

$$
\varepsilon-\operatorname{pmin}(H) \subset \varepsilon-\min (H) \subset \varepsilon-w \min (H)
$$

Proposition 2.3.

Consider a sequence $\left\{\varepsilon_{n} \in C: n \in N\right\}$ decreasing to $\varepsilon \in C$.

Then

$$
\varepsilon-\min (H) \subset \cap\left\{\varepsilon_{n}-\min (H): n \in N\right\} \subset \varepsilon-w \min (H)
$$

and

$$
\cap\left\{\varepsilon_{n}-w \min (H): n \in N\right\}=\varepsilon-w \min (H)
$$

Now the definition of the convex vector valued minimization problem and the corresponding vector valued Lagrangian follows. Then we recall the relationships between $\varepsilon$-solutions of the minimization problem and $\varepsilon$-saddle points of the Lagrangian.

Definition 2.3.

Let

$$
f: X \rightarrow Y \text { and } g: X \rightarrow Z
$$


be proper $C$-convex (and $K$-convex, respectively) functions with $\Delta=d o m \rho \cap d o m g \neq \phi$. We define the minimization problem (MP) as follows

$$
\varepsilon \text {-minimize } \rho(F)
$$

where $F \subset X$ is the feasibility set of the problem $(M P)$ defined by the equality

$$
F=\{x \in X: x \in \Delta, g(x) \leqq 0\} \text {. }
$$

As we already pointed it out, the case $\varepsilon=0$ represents the solutions in the usual (exact) sense.

The Lagrangian of the minimization problem (MP)

$$
\Phi: \Delta \times L^{+}(Z, Y) \rightarrow Y \text {. }
$$

is defined by the equality

$$
\Phi(x, R)=\rho(x)+R \cdot g(x)
$$

The element $\left(x_{0}, R_{0}\right) \in X \times L(Z, Y)$ is an $\varepsilon$-saddle point for the Lagrangian $\Phi$ if the following is met:

(a) $\Phi\left(x_{0}, R_{0}\right) \in \varepsilon-\min \left\{\Phi\left(x, R_{0}\right) \subseteq Y: x \in \Delta\right\}$

(b) $\Phi\left(x_{0}, R_{0}\right) \in \varepsilon-\max \left\{\Phi\left(x_{0}, R\right) \in Y: R \in L^{+}(Z, Y)\right\}$.

Definition 2.4.

We say that the Slater condition holds for the problem $(M P)$ if there exists an $x_{1} \in \Delta$ with $g\left(x_{1}\right)<0$.

Theorem 2.1 .

The element $\left(x_{0}, R_{0}\right) \in X \times L(Z, Y)$ is a $\varepsilon$-saddle point of the Lagrangian $\Phi$, iff

(a) $\Phi\left(x_{0}, R_{0}\right) \in \varepsilon-\min \left\{\Phi\left(x, R_{0}\right) \in Y: x \in \Delta\right\}$

(b) $x_{0} \in F$

(c) $-\varepsilon \neq R_{0} \cdot g\left(x_{0}\right) \leqq 0$.

The property stated in Theorem 2.1. is as much negative as positive. Point (c), namely, turns into the well-known complementarity condition $R_{0} \cdot g\left(x_{0}\right)=0$ in the case of exact saddle points. When $\varepsilon \neq 0$, it only means that

$$
R_{0} \cdot g\left(x_{0}\right) \in(-C \backslash\{-\varepsilon-C\}) \cup\{-\varepsilon\},
$$


where the right hand side is an unbounded set.

Theorem 2.2.

Suppose that the point $\left(x_{0}, R_{0}\right) \in X \times L(Z, Y)$ is a $\varepsilon$-saddle point of the Lagrangian $\Phi$. Then $x_{0} \in X$ is an $\left(\varepsilon-R_{0} \cdot g\left(x_{0}\right)\right)$-solution of the minimization problem (MP).

For the approximation error $\varepsilon-R_{0} \cdot g\left(x_{0}\right) \in C$ we have $0 \leq \varepsilon-R_{0} \cdot g\left(x_{0}\right) \geq 2 \cdot \varepsilon$ as a consequence of the point (c) in Theorem 2.1. However, unlike the scalarized case, transitivity for the relation of non-domination does not hold, and so we cannot claim that $x_{0} \in X$ is a $(2 \cdot \varepsilon)$-solution.

Theorem 2.3.

Suppose that for the problem $(M P)$ the Slater condition holds. If $x_{0} \in X$ is a proper $\varepsilon$-solution of the problem $(M P)$ then there exist an operator $R_{0} \in L^{+}(Z, Y)$ such that $\left(x_{0}, R_{0}\right) \in \Delta \times L_{L}{ }^{+}(Z, Y)$ is an $\varepsilon$-saddle point of the Lagrangian $\Phi$. 


\section{PERTURBATION MAP AND DUALITY}

The procedure that we shall follow is standard. We start by defining a parametrized family of problems (the family of perturbed problems) that includes our original minimization problem. The primal map will then be defined as a function taking the optimal elements of the perturbed problems as values, while for the dual map this will happen via the Lagrangian. Vector minimization problems usually do not have unique solutions in the exact case and this is even more so now. Therefore these functions will be set valued maps.

We shall not reiterate the analogies to the known results in exact vector optimization or scalar $\varepsilon$-optimization but we should like to call for special attention to this issue.

In this section we shall assume that

(i) $f$ is $C$-continuous and $g$ is $K$-continuous,

(ii) $\Delta \subset X$ is compact,

(iii) $\operatorname{int}\left(C^{+}\right) \neq \phi$.

We define for each $u \in Z$

$$
F(u)=\{x \in \Delta: g(x) \leqq u\}
$$

and

$$
Y(u)=\{y \in Y: y=f(x), x \in F(u)\}
$$

As is well known, under our assumptions, $F(u) \subset X$ is a convex set and $Y(u) \subset Y$ is $C$ convex. Furthermore in the case when $u=0, F(u)$ and $Y(u)$ coincides with $F$ and $f(F)$ respectively. Hence the following definition of the perturbed problems $\left(P_{u}\right)$ really means embedding $(M P)$ in a parametrized set of problems:

\section{Definition 3.1 .}

We define the perturbed problems as follows

$$
\varepsilon-\text { minimize } Y(u)
$$

and we call the set valued map defined by the equality

$$
W_{\varepsilon}(u)=\varepsilon-\min \{Y(u)\}
$$

the primal (or $\varepsilon$-primal) map. 
Now we state the basic properties of the primal mapping.

Proposition 3.1.

The equality

$$
W_{\varepsilon}(u)+C=Y(u)+C \quad \forall u \in Z
$$

holds and so, $W_{\varepsilon}$ is a $C$-convex set valued $C$-convex map.

Proof.

The equality is a consequence of Proposition 5.2.1. of Y. Sawaragi, H. Nakayama and T. Tanino [G]. From this and the $C$-convexity of the mapping $Y($.$) the whole$ statement follows.

Proposition 3.2.

Suppose that $\varepsilon_{1} \leqq \varepsilon_{2}$ and $u_{1} \leqq u_{2}$. Then we have

$$
W_{\varepsilon_{1}}(u) \subset W_{\varepsilon_{2}}(u)
$$

and

$$
W_{\varepsilon}\left(u_{1}\right) \subset W_{\varepsilon}\left(u_{2}\right)+C
$$

i. e. the primal map is monotonous in $\varepsilon$ and $C$-monotonous in the variable $u$.

Proof.

The first inclusion follows from Proposition 2.1. On the other hand the monotonicity of the mapping $Y($ ) together with Proposition 3.1. imply

$$
W_{\varepsilon}\left(u_{1}\right) \subset Y\left(u_{1}\right) \subset Y\left(u_{2}\right) \subset W_{\varepsilon}\left(u_{2}\right)+C
$$

Now turn to the dual map and shall use the construction originating from $T$. Tanino and Y. Sawaragi.

Definition 3.2.

Let for each $R \in L^{+}(Z, Y)$ be

$$
\Omega(R)=\{\Phi(x, R) \in Y: x \in \Delta\}
$$

then we define the dual (or $\varepsilon$-dual) map by the equality 


$$
D_{\varepsilon}(R)=\varepsilon-\min \{\Omega(R)+C\}
$$

and the dual problem as

$$
\varepsilon \text {-maximize } U\left\{D_{\varepsilon}(R): R \in L^{+}(Z, Y)\right\}
$$

Proposition 3.3.

The following relation holds for each $r \in[0,1]$ :

$$
D_{\varepsilon}\left(r \cdot R_{1}+(1-r) \cdot R_{2}\right) \subset r \cdot D_{\varepsilon}\left(R_{1}\right)+(1-r) \cdot D_{\varepsilon}\left(R_{2}\right)+C
$$

and so, the function $D_{\varepsilon}$ is a compact, $C$-concave set valued, $C$-concave map.

Proof.

The map $\Phi(., R)$ is $C$-convex because it is a sum of two $C$-convex functions. In other words this means that $\Omega(R)$ is a $C$-convex set. By the $C$-continuity of the function $f$, the $K$-continuity of $g$ and the compactness of $\Delta \subset X, \Omega(R) \subset X$ we can apply Lemmas 2.5. and 2.4. of D. T. Luc [9] implying first that $\Omega(R) \subset Y$ is $C$-compact and then that

$$
D_{\varepsilon}(R)+C=\Omega(R)+C
$$

Hence we can conclude that $D_{\varepsilon}(R) \subset Y$ is $C$-convex.

The $C$-concavity of the mapping $D_{\varepsilon}$ is implied by the following sequence of relations:

$$
\begin{gathered}
D_{\varepsilon}\left(r \cdot R_{1}+(1-r) \cdot R_{2}\right)=\varepsilon-\min \left\{\Omega\left(r \cdot R_{1}+(1-r) \cdot R_{2}\right)\right\} \subset \\
\subset \varepsilon-\min \left\{\Phi\left(x, r \cdot R_{1}+(1-r) \cdot R_{2}\right): x \in \Delta\right\}= \\
=\varepsilon-\min \left\{r \cdot f(x)+(1-r) \cdot f(x)+r \cdot R_{1} \cdot g(x)+(1-r) \cdot R_{2} \cdot g(x): x \in \Delta\right\} \subset \\
\subset \varepsilon-\min \left\{r \cdot \Phi\left(x, R_{1}\right)+(1-r) \cdot \Phi\left(x, R_{2}\right): x \in \Delta\right\}+C= \\
=\varepsilon-\min \left\{r \cdot \Phi\left(x, R_{1}\right): x \in \Delta\right\}+\varepsilon-\min \left\{(1-r) \cdot \Phi\left(x, R_{2}\right): x \in \Delta\right\}+C= \\
=r \cdot D_{\varepsilon}\left(R_{1}\right)+(1-r) \cdot D_{\varepsilon}\left(R_{2}\right)+C .
\end{gathered}
$$

Proposition 3.4 .

$$
D_{\varepsilon}(R)=\varepsilon-\min \cup\left\{W_{\varepsilon}(u)+R \cdot u: u \in Z\right\} \quad \forall R \in L^{+}(Z, Y)
$$


Proof.

The statement follows from the equality

$$
\Omega(R)+C=\left\{W_{\varepsilon}(u)+R \cdot u: u \in Z\right\} \quad \forall R \in L^{+}(Z, Y)
$$

that we shall prove.

Let first be $y \in \Omega(R)$. Then we have

$$
y=\rho\left(x_{1}\right)+R \cdot u_{1}
$$

where we used the notation $u_{1}=g\left(x_{1}\right)$. This implies

$$
f\left(x_{1}\right) \in W_{\varepsilon}\left(u_{1}\right)+C
$$

because $f\left(x_{1}\right) \in Y\left(u_{1}\right)$. Hence

$$
y \in W_{\varepsilon}\left(u_{1}\right)+C+R \cdot u_{1} \subset\left\{W_{\varepsilon}(u)+R \cdot u: u \in Z\right\}
$$

On the other hand, if $y \in W_{\varepsilon}\left(u_{1}\right)+R \cdot u_{1}$ for some $u_{1} \in Z$ then, by the definition of the primal map,

$$
y-R \cdot u_{1} \in \varepsilon-\min \left\{Y\left(u_{1}\right)\right\}
$$

and consequently there exists a $x_{1} \in \Delta$ such that

$$
y-R \cdot u_{1}=f\left(x_{1}\right), \text { and } g\left(x_{1}\right) \leqq u_{1}
$$

Hence we have

$$
y \geqq \rho\left(x_{1}\right)+R \cdot g\left(x_{1}\right)
$$

or that $y \in \Omega(R)+C$.

We are able now to formulate the weak and the the strong duality theorems for our $\varepsilon$-solutions.

Theorem 3.1 .

The relation

$$
y-\varepsilon \neq \rho(x)
$$

holds for each $x \in F$ and $y \in D_{\varepsilon}(R)$ with $R \in L^{+}(Z, Y)$. 
Proof.

By the definition of the dual map,

$$
y-\varepsilon \neq \rho\left(x_{1}\right)+R \cdot g\left(x_{1}\right) \quad \forall x_{1} \in \Delta
$$

holds and the rest of the conditions ensure $R \cdot g\left(x_{1}\right) \leqq 0$.

Theorem 3.2.

(a) Suppose that for some $x \in F$ and $R \in L^{+}(Z, Y)$ the relation $f(x) \in D_{\varepsilon}(R)$ holds. Then $x \in X$ is an $\varepsilon$-solution for the problem $(M P)$ and $f(x) \in Y$ is an $\varepsilon$-maximal element of the $\varepsilon$-dual problem $\left(D_{\varepsilon}\right)$.

(b) Suppose that for the problem $(M P)$ the Slater condition holds and $x \in X$ is a proper $\varepsilon$-solution of $(M P)$. Then $f(x) \in Y$ is an $\varepsilon$-maximal element of the $\varepsilon$-dual problem $\left(D_{\varepsilon}\right)$.

Proof.

We prove (a) by contradiction. If there exists an $x_{1} \in F$ such that $f(x)-\varepsilon \geq f\left(x_{1}\right)$ then we also have

$$
f(x)-\varepsilon \geq f\left(x_{1}\right)+R \cdot g\left(x_{1}\right)
$$

contradicting to the assumptions. To see the rest, let us suppose that there exists an $R_{1} \in L^{+}(Z, Y)$ and a $y \in D_{\varepsilon}\left(R_{1}\right)$ with the property that

$$
f(x)+\varepsilon \leq y .
$$

This implies again

$$
f(x)+R_{1} \cdot g(x)+\varepsilon \leq y
$$

that contradicts to the assumption on $y \in Y$.

We start the proof of (b) by using Theorem 2.3. to establish the existence of an $R \in L^{+}(Z, Y)$ with the property that $f(x) \in D_{\varepsilon}(R)$. Now (a) can be applied and this completes the proof.

Notice that the problem arising in Theorem 2.1. does not appear here, only because we use assumptions that are stronger than the $\varepsilon$-saddle point property in the case when $\varepsilon \neq 0$. 


\section{CONICAL SUPPORTS}

In this section we give a geometric interpretation of the duality and saddle point theorems and summarize our results in one sequence of equivalent statement. This will clearly show where do we have the analogies as expected and where do peculiarities arise.

The fact that the function $W_{\varepsilon}$ takes values among subsets of an ordered vector space implies that the appropriate notion of epigraph is to be defined in the following way:

$$
\text { epi } W_{\varepsilon}=\left\{(u, y) \in Z \times Y: y \in W_{\varepsilon}(u)+C, u \in Z\right\}
$$

By Proposition 3.2., of course, here we have the equality epi $W_{\varepsilon}=\operatorname{epi} Y($.$) . We$ expect that passing from exact solutions to $\varepsilon$-solutions and from scalar values to vector values means the change from a supporting hyperplane to an ' $\varepsilon$-supporting' translate of a cone. This is indeed so. Given an operator $R \in L^{+}(Z, Y)$ let us, namely, define a cone $M_{R}$ in the product space $Z \times Y$ as follows:

$$
M_{R}=\{(u, y) \in Z \times Y: y+R \cdot u \leqq 0\}
$$

and let us denote its linearity space of by $l\left(M_{R}\right)$, that is let be

$$
l\left(M_{R}\right)=\left(-M_{R}\right) \cap M_{R}
$$

This cone is closely related to the structure of $Y$ and $Z$, and has the regularity properties formulated in the following proposition.

Proposition 4.1.

For each $R \in L^{+}(Z, Y), M_{R} \subset Z \times Y$ is a closed convex cone such that

$$
M_{R}=\{0\} \times(-C)+l\left(M_{R}\right) \text {. }
$$

where the lineality space of $M_{R}, l\left(M_{R}\right) \subset Z \times Y$, is isomorphic to the space $Z$.

Proof.

Because of the pointedness of the cone $C \subset Y$ we have that $(u, y) \in L\left(M_{R}\right)$ if and only if $y=-R \cdot u$. Now take any pair $(u, y) \in Z \times Y$ and consider the representation

$$
(u, y)=(0, y+R \cdot u)+(u,-R \cdot u) \text {. }
$$

Easy calculations show that 


$$
-C=\left\{y+R \cdot u \in Y:(u, y) \in M_{R}\right\}
$$

on one hand and that the mapping

$$
I: Z \rightarrow L\left(M_{R}\right)
$$

defined by

$$
I(u)=(u,-R \cdot u)
$$

is an isomorphism.

Definition 4.1.

We say that the cone $M \subset Z \times Y \varepsilon$-supports the set valued map

$$
h: Z \Rightarrow Y
$$

at the point $(u, y) \in \operatorname{graph} h$ if

$$
(-M)+\{(u, y-\varepsilon)\} \cap \operatorname{epi} h \subset\{(u, y-\varepsilon)\}
$$

Analogously, the hyperplane $H \subset Z \times Y \varepsilon$-supports the map $h$ at $(u, y) \in g r a p h h$ if

$$
H+\{0\} \times(-C)+\{(u, y-\varepsilon)\} \cap \text { epi } h \subset\{(u, y-\varepsilon)\}
$$

Let us take now $z^{*} \in Z$ and $y^{*} \in Y$ and $r \in \mathbb{R}$, defining a hyperplane in $Z \times Y$, as follows:

$$
H\left(z^{*}, y^{*}, r\right)=\left\{(u, y) \in Z \times Y:\left\langle y^{*}, y\right\rangle+\left\langle z^{*}, u\right\rangle=r, u \in Z, y \in Y\right\}
$$

In particular, we use the following notation for the $\varepsilon$-supporting hyperplanes of epi $W_{\varepsilon}$ :

$$
H\left(z^{*}, y^{*}\right)=H\left(z^{*}, y^{*}, r_{0}\right)
$$

where

$$
r_{0}=\sup \left\{r \in \mathbb{R}: H\left(z^{*}, y^{*}, r-\left\langle y^{*}, \varepsilon\right\rangle\right)+\{0\} \times C \supset \operatorname{epi} W_{\varepsilon}\right\}
$$

Now we formulate the relationships between $\varepsilon$-supporting cones and hyperplanes of the perturbation function $W_{\varepsilon}$. 
Proposition 4.2.

(a) Suppose that $H\left(z^{*}, y^{*}\right) \subset Z \times Y$ is an $\varepsilon$-supporting hyperplane of epi $W_{\varepsilon}$ at the point $(u, y) \in \operatorname{graph} W_{\varepsilon}, y^{*} \in \operatorname{Eint}\left(C^{+}\right)$and that for the cone $M_{R} \subset Z \times Y$ the relation $l\left(M_{R}\right) \subset H\left(z^{*}, y^{*}, 0\right)$ holds. Then $M_{R} \subset Z \times Y \varepsilon$-supports epi $W_{\varepsilon}$ at the point $(u, y) \in \operatorname{graph} W_{\varepsilon}$.

(b) Suppose that $M_{R} \subset Z \times Y \varepsilon$-supports epi $W_{\varepsilon}$ at the point $(u, y) \in \operatorname{graph} W_{\varepsilon}$. Then there exists a nonzero vector $y^{*} \in C^{+}$and a hyperplane $H\left(z^{*}, y^{*}, r\right) \subset Z \times Y$ that $\varepsilon$-supports epi $W_{\varepsilon}$ at the point $(u, y) \in$ graph $W_{\varepsilon}$ and for which the relation $l\left(M_{R}\right) \subset H\left(z^{*}, y^{*}, 0\right)$ holds.

Proof.

To prove (a) we reason by contradiction. If $M_{R} \subset Z \times Y$ does not $\varepsilon$-support epi $W_{\varepsilon}$ at the point $(u, y) \in$ graph $W_{\varepsilon}$ then there exists another point $\left(u_{1}, y_{1}\right) \in \operatorname{graph} W_{\varepsilon}$ such that

$$
y-\varepsilon-y_{1}+R \cdot u-R \cdot u_{1} \leq 0
$$

For any $y^{*} \in \operatorname{int}\left(C^{+}\right)$, this implies

$$
\left\langle y^{*}, y-\varepsilon-y_{1}+R \cdot u-R \cdot u_{1}\right\rangle<0 \text {. }
$$

On the other hand, relying on the formula representing the elements of $l\left(M_{R}\right) \subset Z \times Y$, and the assumption $l\left(M_{R}\right) \subset H\left(z^{*}, y^{*}, 0\right)$ we obtain

$$
\left\langle z^{*}, u\right\rangle=\left\langle y^{*}, R \cdot u\right\rangle \quad \forall u \in Z \text {. }
$$

Together with the previous inequality, this implies the relation

$$
\left\langle y^{*}, y-\varepsilon\right\rangle+\left\langle z^{*}, u\right\rangle<\left\langle y^{*}, y_{1}\right\rangle+\left\langle z^{*}, u_{1}\right\rangle
$$

contradicting to the $\varepsilon$-supporting property of the hyperplane $H\left(z^{*}, y^{*}\right) \subset Z \times Y$.

Let us prove now (b). By the assumptions we have that

$$
y-\varepsilon-y_{1}+R \cdot u-R \cdot u_{1} \leqq 0 \quad \forall\left(u_{1}, y_{1}\right) \in \operatorname{epi} W_{\varepsilon}
$$

Consider now the mapping

$$
J: Z \times Y \rightarrow Y
$$

defined by the equality

$$
J(u, y)=y+R \cdot u \text {. }
$$


By the linearity of the map $J$ and Proposition 3.1. we have that $J\left(\operatorname{epi} W_{\varepsilon}\right) \subset Y$ is a convex set, and therefore it can be separated by a positive functional from the cone $J\left(-\left(M_{R}\right)+(u, y-\varepsilon)\right) \subset Y$. If we denote this functional by $y^{*} \in C^{+}$and then, by a reasoning similar to the above, we obtain that the hyperplane $H\left(R^{*} \cdot y^{*}, y^{*},\left\langle y^{*}, y+R \cdot u\right\rangle\right) \subset Z \times Y \quad \varepsilon$-supports epi $W_{\varepsilon}$. The inclusion $l\left(M_{R}\right) \subset H\left(R^{*} \cdot y^{*}, 0\right)$ follows from the construction.

Now we summarize the major results of the previous sections in the form of a set of equivalent statements.

Theorem 4.1.

Consider an element $\left(x_{0}, R_{0}\right) \in \Delta \times L^{+}(Z, Y)$. Then the following statements are equivalent:

(a) The pair $\left(x_{0}, R_{0}\right) \in \Delta \times L^{+}(Z, Y)$ is an $\varepsilon$-saddle point of the Lagrangian $\Phi$.

$$
\begin{aligned}
& \Phi\left(x_{0}, R_{0}\right) \in \varepsilon-\min \left\{\Phi\left(x, R_{0}\right) \in Y: x \in \Delta\right\} \\
& x_{0} \in F \\
& -\varepsilon \neq R_{0} \cdot g\left(x_{0}\right) \leqq 0 .
\end{aligned}
$$

(c) $x_{0} \in \Delta$ is an $\left(\varepsilon-R_{0} \cdot g\left(x_{0}\right)\right)$-solution of the problem $(M P)$ and $\rho\left(x_{0}\right) \in Y$ is an $\left(\varepsilon-R_{0} \cdot g\left(x_{0}\right)\right)$-maximal point for the dual problem $\left(D_{\varepsilon-R_{0} \cdot g\left(x_{0}\right)}\right)$.

(d) $x_{0} \in \Delta$ is an $\left(\varepsilon-R_{0} \cdot g\left(x_{0}\right)\right)$-solution of the problem $(M P)$ and the cone $M_{R_{0}} \varepsilon$ supports epi $W_{\varepsilon}$ at the point $\left(0, f\left(x_{0}\right)\right)$.

Proof.

The equivalence of (a) and (b) is stated in Theorem 2.1.

Let us consider now the implication from (b) to (c). That $x_{0} \in \Delta$ is an $\left(\varepsilon-R_{0} \cdot g\left(x_{0}\right)\right)-$ solution of the problem (MP) follows from Theorem 2.2., and by the definition of the dual map this also means that $f\left(x_{0}\right) \in D_{\varepsilon-R_{0} \cdot g\left(x_{0}\right)}$. Hence Theorem 3.2. can be applied and this yields the rest. To see the reverse implication we note first that $f\left(x_{0}\right) \in D_{\varepsilon-R_{0} \cdot g\left(x_{0}\right)}$ is a reformulation of the first relation of (b). The second relation follows from the fact that $x_{0} \in \Delta$ is an $\left(\varepsilon-R_{0} \cdot g\left(x_{0}\right)\right)$-solution of the problem $(M P)$. To prove the last relation we note first that by the positivity of $R_{0} \in L(Z, Y)$, the inequality $R_{0} \cdot g\left(x_{0}\right) \leqq 0$ holds. We also know that

$$
f\left(x_{0}\right)+\varepsilon-R_{0} \cdot g\left(x_{0}\right) \leq f\left(x_{0}\right)+R_{0} \cdot g\left(x_{0}\right)
$$

because $f\left(x_{0}\right) \in Y$ is an $\left(\varepsilon-R_{0} \cdot g\left(x_{0}\right)\right.$ t-maximal element of the dual problem and so we 
proved the third relation, as well.

(c) implies (d) because their first parts are identical and the second part of (d) is just a reformulation of the first relation in (b). The last part of the argument used to prove the implication from (b) to (c) shows that (d) also implies (c). 


\section{CONCLUSION}

In this paper we developed the analogue of the duality theory in vector optimization, on one hand, and of the scalar valued $\varepsilon$-duality results, on the other, for $\varepsilon$ solutions in vector optimization. The significance of $\varepsilon$-solutions in vector optimization arises from the fact that optimization algorithms often produce such results instead of the exact solutions. Using the above theory we obtain guidance in situations when we want to use duality without knowing the solutions exactly. It appears that the duality relations remain true, in general, but we have to cope with such problems as the increase of the approximation error when we start e. g. from saddle points. 


\section{REFERENCES}

1. KUTATELADZE, S.S., Convex E-Programming, Soviet Mathematical Doklady, Vol. 20, No. 2, pp. 391-393, 1979.

2. LORIDAN P., e-Solutions in Vector Minimization Problems, Journal of Optimization Theory and Applications, Vol. 43, No. 2, pp. 265-276, 1984.

3. VALYI I., A General Maximality Principle and a Fixed Point Theorem in Uniform Space, Periodica Mathematica Hungarica, Vol 16, No. 2, pp. 127-134, 1985.

4. WHITE D. J., Epsilon Efficiency, Journal of Optimization Theory and Applications, Vol. 49, No. 2, pp. 319-337, 1986.

5. VAL,YI I., Approximate Saddle Point Theorems in Vector Optimization, Journal of Optimization Theory and Applications, (to appear)

6. SAWARAGI, Y., NAKAYAMA H., TANINO T., Theory of Multiobjective Optimization, Academic Press, New York, 1985.

7. STRODIOT J.J., NGUYEN V.H., HEUKEMES, N., e-Optimal Solutions in Nondifferentiable Convex Programming and Some Related Questions, Mathematical Programming, Vol. 25, pp. 307-328, 1983.

8. VALYI I., Strict Approximate Duality in Vector Spaces, optimization, (to appear).

9. LUC D. T., On Duality Theory in Multiobjective Programming Journal of Optimization Theory and Applications, Vol. 43, No. 4, pp. 557-582, 1984.

10. JAHN J., Duality in Vector Optimization, Mathematical Programming, Vol. 25, pp. 343-353, 1983.

11. NAKAYAMA H., Geometric Consideration of Duality in Vector Optimization, Journal of Optimization Theory and Applications, Vol. 44, pp. 625-655, 1984. 